\title{
Upregulated Inc-HRK-2:1 prompts nucleus pulposus cell senescence in intervertebral disc degeneration
}

\author{
DONGBO LIANG $^{1 *}$, DINGGANG HONG ${ }^{1 *}$, FUYU TANG $^{1}$, YUAN WANG $^{2}$, \\ JIANFENG LI ${ }^{1}$, LINQING LI ${ }^{1}$ and HUAMING CHEN ${ }^{1}$ \\ ${ }^{1}$ Department of Orthopaedics, Liuzhou Traditional Chinese Medical Hospital, Guangxi University of Chinese Medicine, \\ Liuzhou, Guangxi 545001; ${ }^{2}$ The Second Department of Orthopaedic Surgery, Changzheng Hospital, \\ Second Military Medical University, Shanghai 200003, P.R. China
}

Received February 7, 2020; Accepted May 28, 2020

DOI: $10.3892 / \mathrm{mmr} .2020 .11603$

\begin{abstract}
Intervertebral disc (IVD) degeneration is a complicated physiological change involving cellular senescence, inflammation and the degradation of the extracellular matrix. Long non-coding RNAs (lncRNAs) have been identified as new players in IVD degeneration. The present study aimed to identify lncRNAs implicated in IVD degeneration via the regulation of cellular senescence. In the present study, nucleus pulposus (NP) cells isolated from moderately degenerated IVD tissues exhibited a senescent phenotype with increased senescence rates, detected by senescence-associated $\beta$-galactosidase (SA- $\beta$-gal) staining, and reduced growth and migratory abilities. Microarray and target prediction analyses identified 353 differentially expressed lncRNAs, and 251 cis- and 2,170 trans-acting targets in degenerated NP cells. Bioinformatic analyses revealed that these predicted targets were enriched in the regulation of response to DNA damage stimulus, positive regulation of cell cycle processes and interferon- $\beta$ production. In addition, a network of the top 10 upregulated and top 10 downregulated IncRNA targets was constructed, and two trans-acting targets, $\mathrm{C}-\mathrm{C}$ motif chemokine ligand 5 (CCL5) and polyribonucleotide nucleotidyltransferase 1 ( $P N P T 1)$ involved in aging or senescence, and their corresponding lncRNAs,
\end{abstract}

Correspondence to: Dr Fuyu Tang, Department of Orthopaedics, Liuzhou Traditional Chinese Medical Hospital, Guangxi University of Chinese Medicine, 32 North Jiefang Road, Liuzhou, Guangxi 545001, P.R. China

E-mail: chinastrongman@126.com

Dr Yuan Wang, The Second Department of Orthopaedic Surgery, Changzheng Hospital, Second Military Medical University, 415 Fengyang Road, Shanghai 200003, P.R. China

E-mail: 13701948727@163.com

${ }^{*}$ Contributed equally

Key words: lnc-HRK-2:1, cellular senescence, intervertebral disc degeneration, C-C motif chemokine ligand 5, polyribonucleotide nucleotidyltransferase 1
lnc-ST8SIA5-1:2 and lnc-HRK-2:1, were identified. Reverse transcription-quantitative PCR validation demonstrated that the two targets and two candidate IncRNAs were significantly upregulated in degenerated NP cells. Overexpression of lnc-HRK-2:1, with validated higher expression levels, in normal NP cells induced a senescent phenotype, with enhanced rates of senescence detected by SA- $\beta$-gal staining in cells, decreased growth and migratory abilities and improved expression levels of CCL5 and PNPT1. Collectively, these results suggested that upregulation of lnc-HRK-2:1 prompted NP cell senescence in IVD degeneration, which may be associated with increased expression levels of CCL5 and PNPT1.

\section{Introduction}

Intervertebral disc (IVD) degeneration is a common aging-related physiological change and is mostly a non-morbid condition (1). However, lower back pain caused by IVD degeneration brings long-term economic burdens for societies and families (2). The normal physiological functions of IVDs are dependent on the central highly-hydrated nucleus pulposus (NP) containing considerable amounts of proteoglycans and aggrecans, as well as the outer annulus fibrosus (2). Once IVD occurs with aging-related or pathological damage-induced degeneration, a series of alterations to the IVD microenvironment can be observed, such as imbalances in extracellular matrix (ECM) metabolism, reduced hydration, increased inflammatory cytokine expression levels, as well as accumulating cellular senescence $(3,4)$. These alterations are closely associated, and interactions among them can aggravate the rate of degeneration (4).

Cell senescence is an irreversible cell-cycle arrest that occurs in response to various external stimuli (5). Previous studies have demonstrated that the expression of senescence-associated $\beta$-galactosidase (SA- $\beta$-gal), a marker of senescence in IVD cells, is positively associated with IVD pathological grading $(6,7)$. NP cells isolated from degenerated IVDs exhibit slower proliferation and increased cellular senescence compared with those from non-degenerated IVD $(8,9)$. In addition, these senescent IVD cells with a low rate of proliferation are metabolically viable and exhibit altered expression of various catabolic cytokines and degradative 
enzymes, which further aggravates the imbalances in ECM metabolism, as well as reduces the hydration status in IVD (1). These observations emphasize the significant role of cellular senescence in the development of IVD degeneration. To date, pathways such as p53-p21-retinoblastoma protein (RB) and p16INK4a-RB have been reported to contribute to degeneration of the IVD $(1,10)$; however, the underlying molecular mechanisms remain unclear.

Long non-coding RNAs (lncRNAs), first identified in 1991 from cDNA, are RNA transcripts $>200$ nucleotides long that lack evident open reading frames (11). LncRNAs are widely expressed in tissues and cells, and their expression patterns exhibit some specificity (12). Studies have reported that lncRNAs regulate gene expression via their interactions with other RNAs or proteins, in spite of lacking protein-coding capacity; consequently, lncRNAs serve various roles in cellular, physiological or pathological processes including cellular senescence $(13,14)$. Although a number of lncRNAs such as lncPolE and FAM83H-AS1 have been verified as players in IVD degeneration $(15,16)$, the lncRNAs involved in IVD degeneration via the regulation of cell senescence are largely unknown.

Microarray technology is a highly-effective and convenient method to identify differentially expressed (DE) genes or lncRNAs, providing abundant potential choices for mechanisms and candidate target studies (17). Thus, the present study aimed to evaluate cellular senescence of NP cells in patients with moderate IVD degeneration, identify and analyze DE IncRNAs, and confirm the role of cellular senescence-associated lncRNA in IVD degeneration, as well as to identify novel mechanisms and potential therapeutic targets involved in IVD degeneration.

\section{Materials and methods}

Sample collection. A total of 6 NP specimens were obtained from three healthy controls whose IVD was mechanically damaged in car accidents, and three patients with grade III IVD degeneration between March 2017 and June 2018. In the control patients who had undergone mechanical trauma, only those diagnosed with NP herniation and the IVD degeneration confirmed to be grade I before surgery were considered; the herniated NP tissue was resected within 3 days after the accident. The degenerative grade was evaluated by magnetic resonance imaging according to the Pfirrmann grading system (18). Patients with degenerative spinal stenosis, idiopathic scoliosis, tumors, infections or previous lumbar disc surgery were excluded from the study. Ages of the participants (males, 4; females, 2) ranged between 28 and 33 years, and the mean age was 29.3 years. All NP tissue samples were carefully collected under sterile conditions. The present study was approved by the Ethics Review Board of Liuzhou Traditional Chinese Medicine Hospital, and the study was performed in accordance with the Declaration of Helsinki.

Cells and cell culture. NP cell isolation was performed as previously described (19). Briefly, NP tissues were washed with PBS three times and cut into small pieces $\left(1-2 \mathrm{~mm}^{3}\right)$. F-12 complete medium (Gibco; Thermo Fisher Scientific, Inc.), supplemented with $10 \%$ fetal bovine serum (FBS; HyClone; Cytiva) and $1 \%$ penicillin/streptomycin (Invitrogen; Thermo Fisher Scientific, Inc.), was used to culture the tissue pieces at $37^{\circ} \mathrm{C}$ with $5 \% \mathrm{CO}_{2}$.
The sample fragments were removed when NP cells migrated out of the tissues. The remaining NP cells were cultured and amplified in F-12 complete medium. NP cells obtained from specimens from healthy participants were used as a control group, whereas those from patients served as the degeneration group.

Reverse-transcription quantitative PCR ( RT- $q P C R)$. Total RNA was isolated from NP cells using TRIzol ${ }^{\circledR}$ reagent (Invitrogen; Thermo Fisher Scientific, Inc.), and $1 \mu \mathrm{g}$ RNA and random primers were used for cDNA synthesis according to the protocol of the PrimeScript RT reagent kit (TaKaRa Biotechnology Co., Ltd.). SYBR ${ }^{\circledR}$ Premix Ex Taq ${ }^{\mathrm{TM}}$ (TaKaRa Biotechnology Co, Ltd.) was used to determine the selected lncRNA and mRNA expression levels using 7500 Fast Real-Time PCR system (Applied Biosystems; Thermo Fisher Scientific, Inc.) according to the manufacturer's instructions. The reaction was incubated at $95^{\circ} \mathrm{C}$ for $5 \mathrm{~min}$, followed by 42 cycles of $95^{\circ} \mathrm{C}$ for $10 \mathrm{sec}$ and $60^{\circ} \mathrm{C}$ for $60 \mathrm{sec}$. The specific primers used were procured from Sangon Biotech Co., Ltd., and their sequences are listed in Table I. GAPDH was used as a normalization control for lncRNAs and mRNAs. Data were analyzed using the $2^{-\Delta \Delta \mathrm{Cq}}$ method (20).

SA- $\beta$-gal staining assay. The number of senescent NP cells was determined using a SA- $\beta$-gal staining kit (Beyotime Institute of Biotechnology) according to the manufacturer's instructions. Briefly, $3 \times 10^{5}$ cells/well were seeded into 6-well plates. When confluency reached $\sim 80 \%$, the cells were fixed with $1 \mathrm{ml}$ fixative solution for $15 \mathrm{~min}$ at room temperature, washed three times with PBS and stained with $1 \mathrm{ml}$ specific working solution overnight at $37^{\circ} \mathrm{C}$. The next day, five random visual fields (magnification, $\mathrm{x} 400$ ) were captured by light microscopy and the the rate of SA- $\beta$-gal-positive cells was calculated as the following equation: SA- $\beta$-gal-positive cells rate $=\mathrm{SA}-\beta$-gal-positive cell number $/$ total cell number $\mathrm{x} 100 \%$.

Viability assay. A total of $4 \times 10^{3} \mathrm{NP}$ cells/well were seeded into 96-well plates. Following culture for 1, 3, 5 and 7 days, $15 \mu 1$ MTT solution (Sigma-Aldrich; Merck KGaA) was added to each well, and the cells were incubated for a further $4 \mathrm{~h}$ at $37^{\circ} \mathrm{C}$. Then, $100 \mu$ ldimethyl sulfoxide (Sigma-Aldrich; Merck KGaA) was added to dissolve the formazan crystals. Absorbance of the samples was detected at $492 \mathrm{~nm}$ using a microplate reader (Bio-Rad Laboratories, Inc.).

Migration assay. Transwell chamber inserts in 24-well plates (pore size, $8 \mu \mathrm{m}$; Corning Life Sciences) were used for NP cell migratory ability evaluation. Briefly, $4 \times 10^{4}$ cells in $100 \mu 1$ serum-free DMEM (Gibco; Thermo Fisher Scientific, Inc.) were carefully added to the upper chamber, and $600 \mu \mathrm{l}$ complete DMEM (10\% FBS; Gibco; Thermo Fisher Scientific, Inc.) was added to the lower chamber in order to induce migration. Following incubation for $20 \mathrm{~h}$ at $37^{\circ} \mathrm{C}$, the cells in the upper chamber were gently removed, and those that had migrated to the lower chamber were fixed with $100 \%$ methanol (Sigma-Aldrich; Merck KGaA) for 10 min and stained with $0.1 \%$ crystal violet (Sigma-Aldrich; Merck KGaA) for another $10 \mathrm{~min}$ at room temperature. Finally, stained cells in three random visual fields (magnification, x200) were captured under a light microscope 
Table I. Primer sequences for lncRNAs and genes.

\begin{tabular}{ll}
\hline Target & \multicolumn{1}{c}{ Sequences $\left(5^{\prime} \rightarrow 3^{\prime}\right)$} \\
\hline lnc-ST8SIA5-1:2 & F: GGAAACCTTTTGCCCTGGAG \\
& R: TGAGAGGAAAGCAAGGGAGG \\
Inc-HRK-2:1 & F: AGGACACGGGAAGCTTTTCT \\
& R: CCAACAACGTCAGAACCCAG \\
CCL5 & F: CCAGCAGTCGTCTTTGTCAC \\
& R: CTCTGGGTTGGCACACACTT \\
PNPT1 & F: GCGAGCACTATGGAGTAGCG \\
& R: GCAGTGTCACCTGACTGTACTA \\
GAPDH & F: GGAGCGAGATCCCTCCAAAAT \\
& R: GGCTGTTGTCATACTTCTCATGG
\end{tabular}

Lnc, long non-coding RNA; CCL5, C-C Motif Chemokine Ligand 5; PNPT1, polyribonucleotide nucleotidyltransferase 1; F, forward; R, reverse.

and counted with ImageJ software (version 1.8.0; National Institutes of Health).

Microarray analysis. Total RNA was extracted using TRIzol ${ }^{\circledR}$ reagent (Invitrogen; Thermo Fisher Scientific, Inc.) and sent to Shanghai Biotechnology Corporation to complete the microarray analysis. The RNeasy micro kit (Qiagen $\mathrm{GmbH}$ ) and RNase-Free DNase set (Qiagen $\mathrm{GmbH}$ ) were used to purify the total RNA according to the manufacturer's instructions. The purified RNA was labeled and amplified by Low Input Quick Amp Labeling kit (Agilent Technologies, Inc.) according to the manufacturer's instructions. Subsequently, $1.65 \mu \mathrm{g}$ Cy3-labeled cRNA of each sample was loaded onto a custom microarray for hybridization, and microarray scanning was performed on an Agilent Microarray Scanner (Agilent Technologies, Inc.). The resulting data were extracted with Feature Extraction software 10.7 (Agilent Technologies, Inc.), and the raw data were processed using the $\mathrm{R}$ statistical software package (version 3.4.1) (21). DE lncRNAs were filtered following the criteria absolute fold-change $[\mathrm{FC}$ (abs) $] \geq 2$ and $\mathrm{P}<0.05$.

Prediction of IncRNA targets. It has been suggested that IncRNAs regulate gene expression in the cis- and trans-regulatory manner $(22,23)$. In the present study, genes transcribed within $10 \mathrm{~kb}$ upstream or downstream of the DE lncRNAs were considered as cis-targets. LncRNAs and their potential cis-targets were paired and visualized using the UCSC genome browser (genome.ucsc.edu/) (24). For trans-regulation prediction, two criteria were used for screening: i) Sequence complementarity of DE lncRNAs and potential mRNA targets, and ii) the complementary energy between the two sequences was $\leq-30$. BLAST software (National Center for Biotechnology Information) was used for the first screening, while RNAplex software (bioinf.uni-leipzig.de/Software/RNAplex/) was used to decide on the trans-acting targets by the calculation of complementary energy.

Bioinformatics analyses. Gene Ontology (GO) enrichment analysis (geneontology.org) (25) and Kyoto Encyclopedia of
Genes and Genomes (KEGG) pathway analysis (genome.jp/ $\mathrm{kegg} /$ ) (26) were performed to assess the potential roles of the lncRNA targets. Gene number $\geq 2$ and $\mathrm{P}<0.05$ were used as thresholds to screen relevant GO terms and KEGG pathways.

Cell transfection. NP cells from the control group were seeded in 6 -well plates $\left(2 \times 10^{5}\right.$ cells/well). When confluence reached $\sim 80 \%$, the cells were transfected with $4 \mu \mathrm{g}$ pCDH-CMV-MCS-EF1-GFP-T2A-Puro plasmid (Hanbio Biotechnology Co., Ltd.) encoding the full length of lnc-HRK-2:1 or equivalent empty vector using Lipofectamine ${ }^{\circledR}$ 2000 (Invitrogen; Thermo Fisher Scientific, Inc.) according to the manufacturer's instructions. Transfection was performed for $48 \mathrm{~h}$ at $37^{\circ} \mathrm{C}$, and the resulting cells were harvested for RT-qPCR validation. NP cells transfected with the plasmid or empty vector were termed lnc-HRK-2:1 or negative control (NC) groups, respectively.

Statistical analysis. Statistical analysis was performed using SPSS 17.0 software (SPSS Inc.). Data are presented as the mean \pm standard deviation. Unpaired Student's t-test was used to analyze the differences between groups. $\mathrm{P}<0.05$ was considered to indicate a statistically significant difference.

\section{Results}

Phenotypic differences of control and degenerated NP cells. As presented in Fig. 1A, control NP cells were long in shape with small nuclei. The majority of degenerative NP cells were morphologically similar to the control cells, whereas a minority were short shuttle-like, polygonal or rounded in shape. SA- $\beta$-gal staining results indicated that the number of SA- $\beta$-gal-positive cells was significantly higher in the degenerated group compared with that in the control group (Fig. 1B and C). In addition, the abilities of the cells to grow and migrate were significantly decreased in degenerated NP cells compared with those of the control cells (Fig. 1D-F). These results indicated that degenerative NP cells exhibited increased cellular senescence and reduced growth and migratory abilities.

Identification of DE lncRNAs in degenerated NP cells. LncRNA microarray analysis screened and identified 353 DE IncRNAs that satisfied the $\mathrm{FC} \geq 2$ and $\mathrm{P}<0.05$ thresholds between the control and degenerated NP cells (Table SI). Heatmaps (Fig. 2A) provided a clear outline of lncRNA expression in each sample, and the general expression trends of IncRNAs from three independent assays in each group were relatively consistent. Among the 353 DE lncRNAs, 228 were downregulated and 125 were upregulated, presented as blue and red dots, respectively, in Fig. 2C. The most upregulated lncRNA was NR_026812, and its FC was close to the FC (abs) of ENST00000438810, which was the most downregulated IncRNA. The top 10 upregulated and top 10 downregulated IncRNAs are listed in Tables II and III.

Prediction of DE lncRNA targets. For improved understanding of the unknown lncRNAs, the present study predicted the potential cis- and trans-targets of the DE IncRNAs, which may mediate the expression of nearby genes located on the same 
A
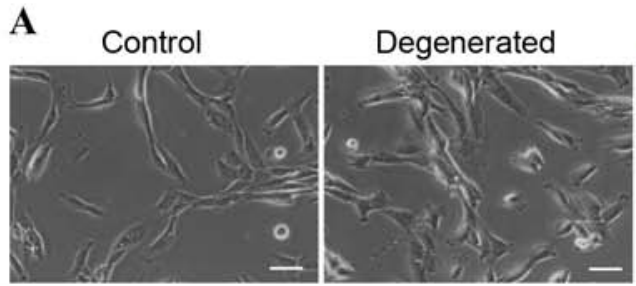

D

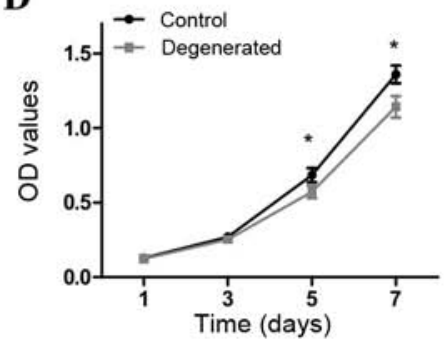

$\mathbf{E}$
B

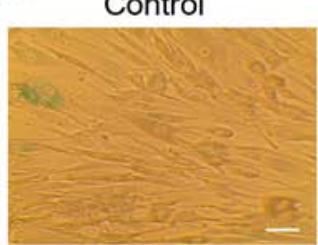

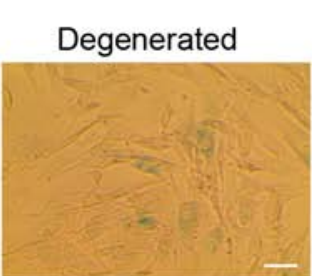

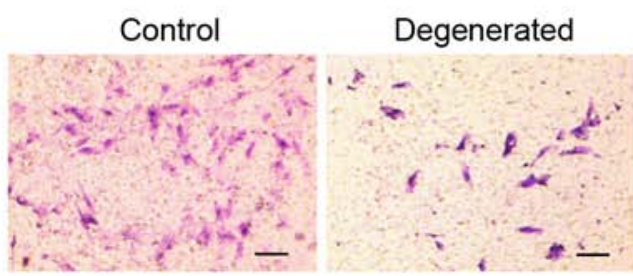

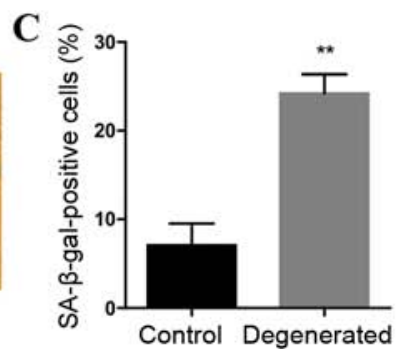

F

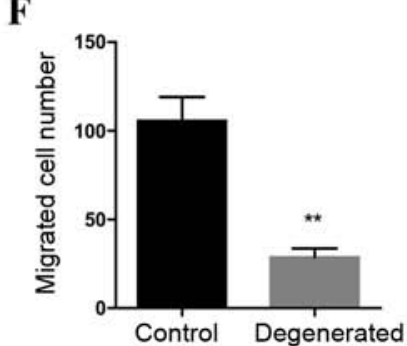

Figure 1. Phenotypic differences between the control and degenerated NP cells. (A) Cell morphology of control and degenerated NP cells. Scale bar, $50 \mu \mathrm{m}$. (B and C) SA- $\beta$-gal staining analysis of senescent NP cells in the control and degenerated groups. Scale bar, $50 \mu \mathrm{m}$. (D) MTT assay was used to evaluate cell viability. (E and F) Transwell assays were performed to detect cell migratory ability. Scale bar, $100 \mu \mathrm{m}$. ${ }^{*} \mathrm{P}<0.05$, ${ }^{* * *} \mathrm{P}<0.01$ vs. control. NP, nucleus pulposus; OD, optical density; SA- $\beta$-gal, senescence-associated $\beta$-galactosidase.

A

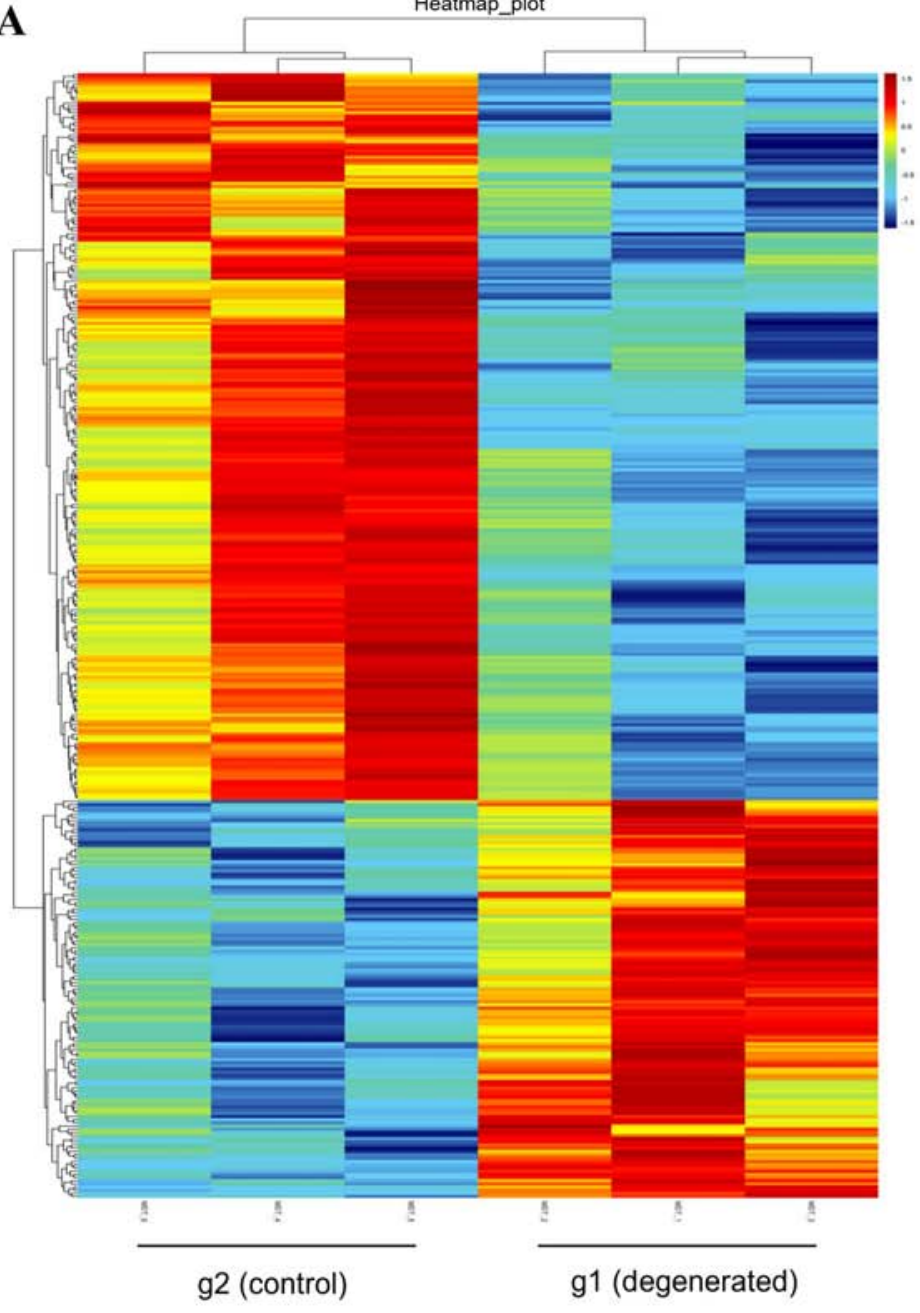

B

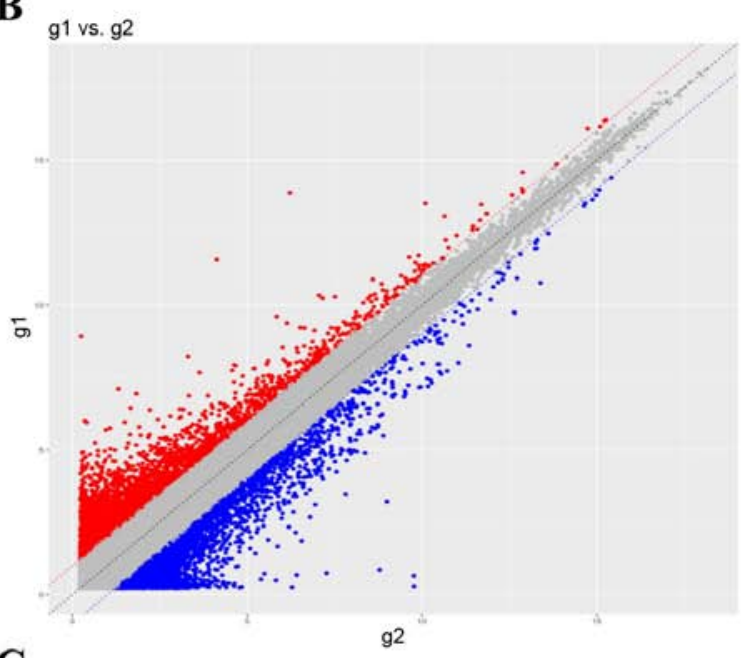

C

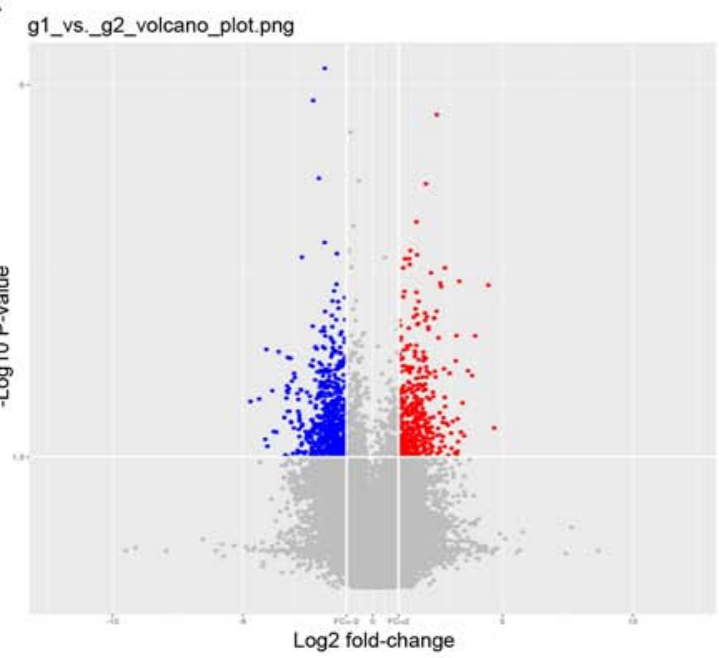

Figure 2. Identification of DE lncRNAs in degenerated NP cells. (A) Heat map of 353 DE lncRNAs in NP cells in the control (g2) and degenerated (g1) groups. (B) Scatter plot of lncRNAs with FC (abs) $\geq 2$. (C) Volcano plot of the 353 DE lncRNAs. The vertical lines represent FC (abs) $=2$ and the horizontal line represents $\mathrm{P}=0.05$. Dots indicate up-(red) and down-(blue) regulated lncRNAs. DE, differentially expressed; NP, nucleus pulposus; FC, fold-change; abs, absolute; lncRNA, long non-coding RNA. 
Table II. Top 10 upregulated lncRNAs in degenerated nucleus pulposus cells.

\begin{tabular}{rllll}
\hline No. & \multicolumn{1}{c}{ LncRNA } & P-value & FC & Database \\
\hline 1 & NR_026812 & 0.0257 & 25.531 & RefSeq \\
2 & ENST00000438158 & 0.0010 & 21.957 & ENSEMBL_GENCODE \\
3 & NR_122111 & 0.0031 & 15.407 & RefSeq \\
4 & ENST00000454588 & 0.0069 & 12.723 & ENSEMBL_GENCODE \\
5 & lnc-HRK-2:1 & 0.0009 & 10.085 & lncipedia \\
6 & lnc-MFAP5-3:2 & 0.0353 & 9.782 & lncipedia \\
7 & NR_031707 & 0.0467 & 9.433 & RefSeq \\
8 & NR_105061 & 0.0031 & 9.382 & RefSeq \\
9 & lnc-ST8SIA5-1:2 & 0.0073 & 7.804 & lncipedia \\
10 & lnc-HFE2-2:1 & 0.0486 & 7.482 & lncipedia
\end{tabular}

FC, fold-change; lnc, lncRNA, long non-coding RNA.

Table III. Top 10 downregulated lncRNAs in degenerated nucleus pulposus cells.

\begin{tabular}{rlllll}
\hline No. & \multicolumn{1}{c}{ LncRNA } & P-value & FC & FC (abs) & Database \\
\hline 1 & ENST00000438810 & 0.0139 & 0.039 & 25.883 & ENSEMBL_GENCODE \\
2 & NR_026576 & 0.0131 & 0.049 & 20.496 & RefSeq \\
3 & lnc-GABRE-2:1 & 0.0333 & 0.057 & 17.458 & lncipedia \\
4 & lnc-TMEM229B-2:1 & 0.0043 & 0.059 & 16.935 & lncipedia \\
5 & lnc-RP11-383H13.1.1-4:1 & 0.0391 & 0.061 & 16.502 & lncipedia \\
6 & ENST00000518064 & 0.0109 & 0.069 & 14.465 & ENSEMBL_GENCODE \\
7 & lnc-TUBB2A-5:1 & 0.0279 & 0.072 & 13.947 & lncipedia \\
8 & lnc-HOXD3-1:6 & 0.0282 & 0.077 & 12.997 & lncipedia \\
9 & lnc-MT1G-2:3 & 0.0045 & 0.082 & 12.210 & lncipedia \\
10 & NR_125920 & 0.0202 & 0.095 & 10.500 & RefSeq \\
\hline
\end{tabular}

FC, fold-change; abs, absolute; lncRNA, long non-coding RNA.

chromosome and genes located on other chromosomes, respectively. As a result, a total of 251 cis- and 2,170 trans-regulatory targets were identified (Tables SII and SIII), and targets of the top 20 DE lncRNAs were included in a lncRNA target network (Fig. 3). Among the 20 lncRNAs, 12 had cis- or trans-targets. Of note, targets of lnc-ST8SIA5-1:2 and lnc-HRK-2:1, sharing almost one-third of the trans-regulatory targets, were abundant compared with the limited targets of the other 10 lncRNAs.

Bioinformatics analyses of predicted lncRNA targets. Bioinformatics analyses of the cis- and trans-regulatory targets were subsequently performed. GO term analysis demonstrated that the majority of the cis- and trans-acting targets were enriched in 'cellular processes' and 'binding' terms (Figs. S1 and S2). The top 30 GO enrichment results provided more details. Cis-acting targets were involved in the 'regulation of response to DNA damage stimulus', 'positive regulation of cell cycle processes' and 'positive regulation of cell cycle arrest, whereas trans-acting targets were associated with 'interferon- $\beta$ production' and 'cellular aldehyde metabolic process' (Fig. 4), which may underlie the involvement of the DE lncRNAs in cellular senescence. KEGG pathway analysis demonstrated that the cis-regulatory targets were implicated in 'apoptosis' and 'proteasome' pathways, and the trans-regulatory targets were enriched in 'lysosome' and 'glycosylphosphatidylinositol (GPI)-anchor biosynthesis' (Figs. S3 and S4).

$R T-q P C R$ validation of senescence-associated lncRNAs and targets. To screen cellular senescence-associated targets of lncRNAs with high FC, key words such as 'aging' and 'senescence' were retrieved in GO and KEGG enrichment analyses, and 15 targets (Table IV) were identified among the trans-targets in GO enrichment. Next, the 15 targets were searched in the network of the top 20 lncRNA targets (Fig. 3) to screen the coincident and cis-acting targets C-C motif chemokine ligand 5 (CCL5) and polyribonucleotide nucleotidyltransferase 1 (PNPT1), and its two lncRNAs, lnc-ST8SIA5-1:2 and lnc-HRK-2:1 were screened out. Then, the expression levels of the two lncRNAs and their two targets were detected. RT-qPCR results demonstrated that the two lncRNAs were significantly upregulated in degenerative NP 


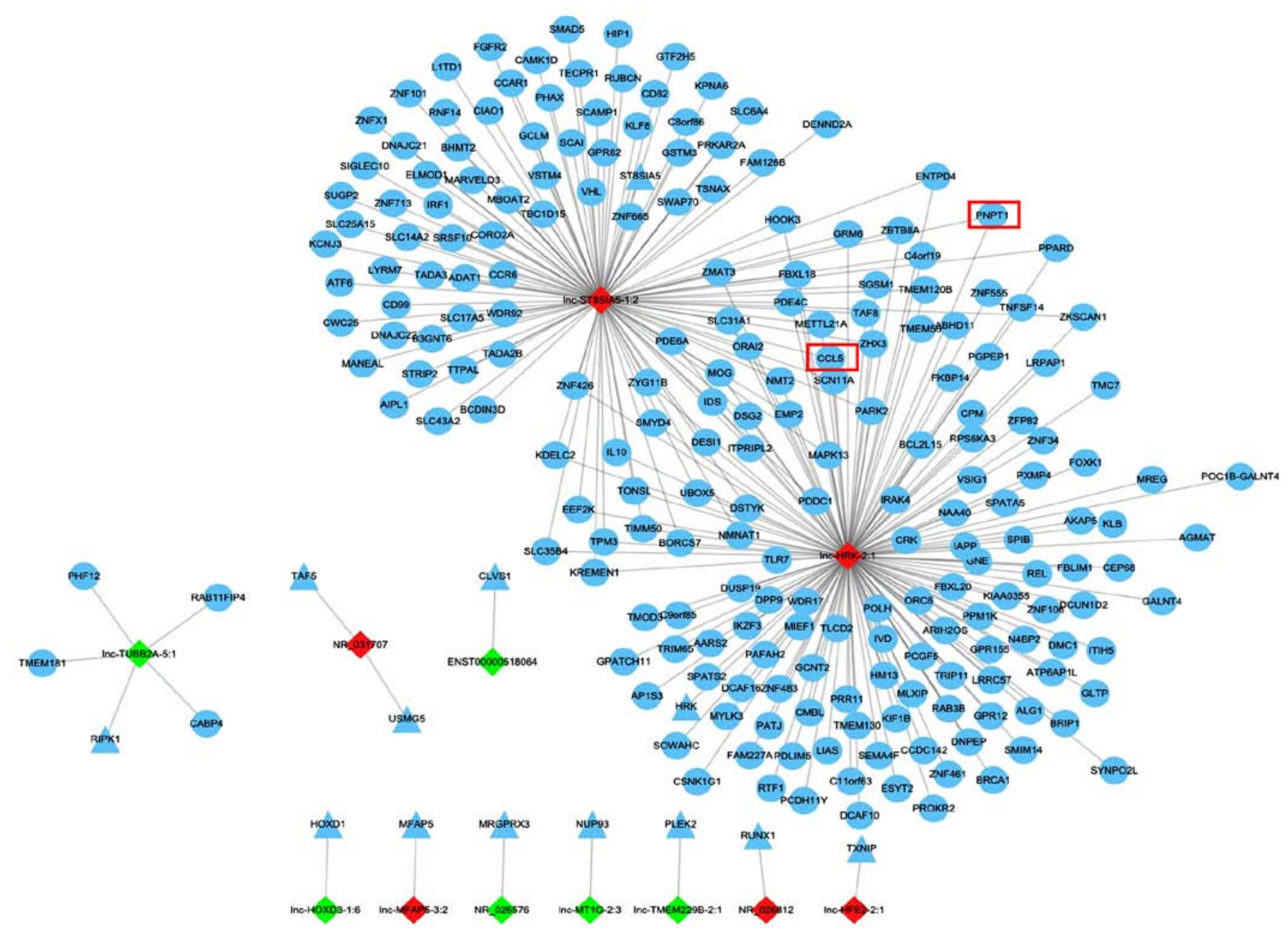

Figure 3. Network of cis- and trans-targets of the top 20 DE lncRNAs. DE lncRNAs are marked with red (upregulated) and green (downregulated) diamonds; blue triangles (cis-) and circles (trans-) denote the regulatory targets. Among the 20 lncRNAs, 12 had cis- or trans-targets. Trans-targets marked with red rectangles (CCL5 and PNPT1) are senescence-associated genes. DE, differentially expressed; IncRNA, long non-coding RNA; CCL5, C-C motif chemokine ligand 5; PNPT1, polyribonucleotide nucleotidyltransferase 1.

cells compared with the control cells, consistent with microarray analysis results (Fig. 5A). In addition, the expression levels of the two targets were enhanced in the degeneration group compared with those in the control group (Fig. 5A).

Overexpression of lnc-HRK-2:1 induces a senescent phenotype of control NP cells. Lnc-HRK-2:1 was selected for further study due to its high expression levels in degenerative NP cells. Lnc-HRK-2:1 was overexpressed in control NP cells, which was validated by RT-qPCR (Fig. 5B). SA- $\beta$-gal staining results demonstrated that the numbers of senescent cells were significantly increased in control NP cells following the overexpression of lnc-HRK-2:1 (Fig. 5C and D). In addition, suppressed growth and migratory abilities were also observed in the lnc-HRK-2:1-overexpressing NC cells compared with those in the NC group (Fig. 5E-G). These altered phenotypes suggested a senescence-inducing effect of lnc-HRK-2:1 on phenotypic transition in control NP cells. The expression levels of the two target genes were also detected, and the results revealed significant upregulation of CCL5 and PNPT1 expression levels in control NP cells following overexpression of lnc-HRK-2:1 compared with those in the NC group (Fig. 5H).

\section{Discussion}

Generally, aging is regarded as a dependent variable to investigate the effects of senescence- or aging-mediated mechanisms for IVD degeneration $(1,9,27)$. However, the onset of IVD degeneration is not solely dependent on aging factors. Interactions between external stimuli and aging or external stimuli-induced cellular senescence are similar to genuine pathological circumstances (5). The present study aimed to screen lncRNAs involved in cellular senescence in IVD degenerative samples to provide a realistic pathological development mechanism.

To exclude the factor of aging, young patients of a similar age were selected as sources of NP tissues in the present study. For the control group, NP tissues were collected from patients who had suffered car accidents, who commonly experience IVD protrusion $(28,29)$. In a vehicle accident, the pressure on the lumbar joints can be abruptly increased, resulting in joint compression, fiber ring rupture and NP herniation, and may cause spinal compression and further motor deficit if herniated NP tissues are not resected in a timely manner (29). In the present study, only the IVD degeneration of patients confirmed to be grade I was used as control samples. 
Table IV. Aging- and senescence-associated target genes.

\begin{tabular}{lllll}
\hline GO ID & \multicolumn{1}{c}{ Term } & Type & Count & \\
\hline 0007569 & Cell aging & BP & 8 & Genes \\
0090398 & Cellular senescence & BP & 4 & ATM PNPT1 \\
0007568 & Aging & BP & 15 & CERF2 SMC5 ZKSCAN3 PNPT1 \\
& & & & TERF2 P2RY2 PNPT1 NQO1 IL15 CASP2 \\
& & & LIMS1 STAT3 ATM
\end{tabular}

BP, biological process; GOID, Gene Ontology identifier.
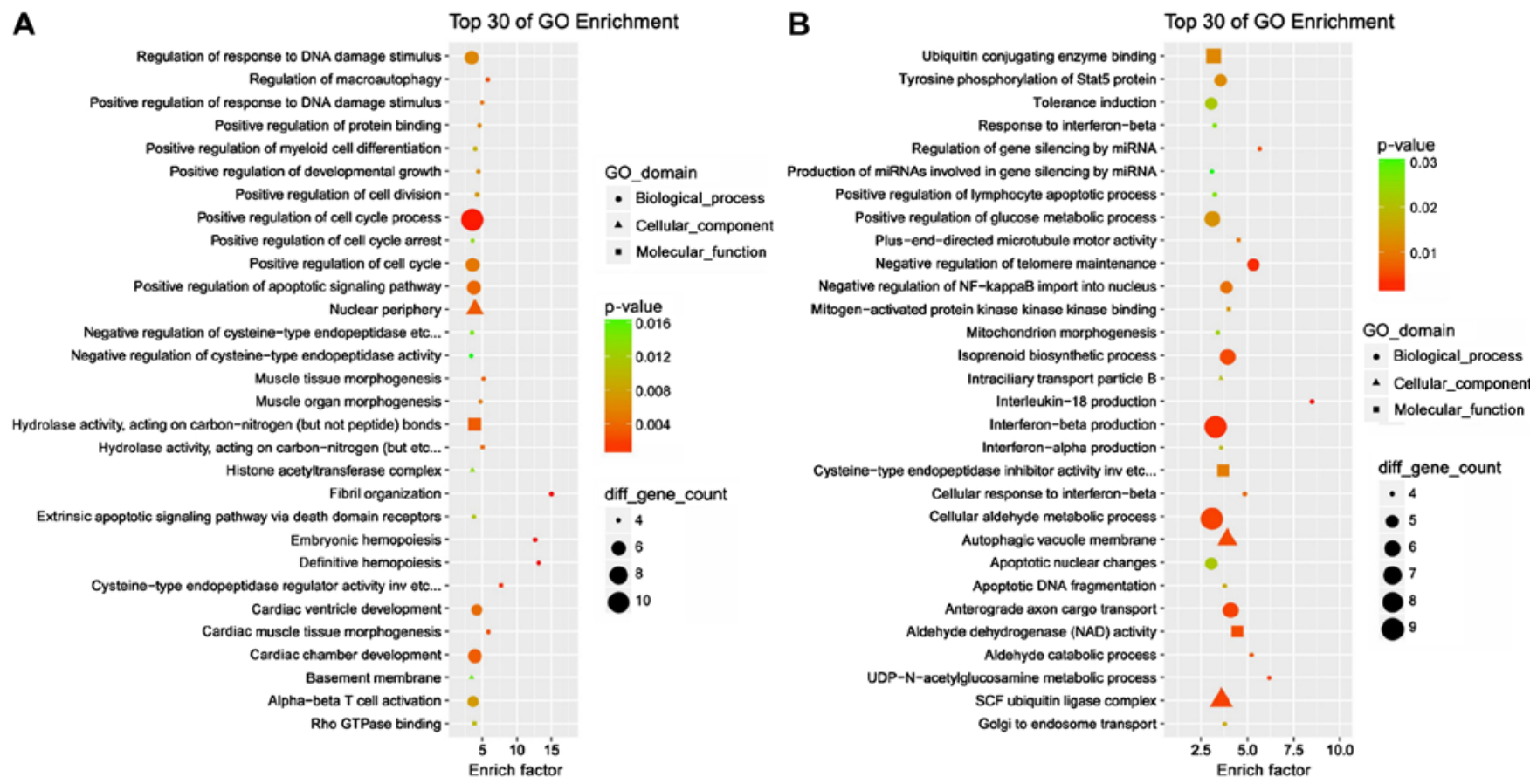

Figure 4. Top 30 GO enriched (A) cis- and (B) trans-targets. GO terms were divided into three classifications, 'biological process', 'cellular component' and 'molecular function', which are graphically distinguished by circles, triangles and squares, respectively. The sizes of the shapes represent the counts of cis- or tans-targets, and the colors indicate the P-values. GO, Gene Ontology.

NP cells isolated from the two groups investigated in the present study were morphologically similar, and only a limited number of the degenerated NP cells were rounded. Generally, IVD cells undergo morphological changes from initially being spindle-shaped and turning rounded during aging and degeneration (30). Similar morphological alterations were also confirmed in NP cells in a rat aging model (9). Thus, the limited number of rounded NP cells from the degeneration group in the present study suggested that slight cellular senescence was occurring in patient NP tissues, which may be attributed to the lower ages and moderate level of IVD degeneration of these patients. Cell phenotyping results revealed that degenerated NP cells exhibited increased cellular senescence and decreased growth and migratory abilities. These phenotypic changes were consistent with previous studies involving models of aging and clinical cases $(9,31)$.

LncRNAs are a class of molecules that serve roles in regulating gene expression and have been demonstrated to be involved in diverse physiological and pathogenic effects in the endocrine, reproductive, metabolic, immune, nervous and cardiovascular systems (32). The present study identified 353 DE IncRNAs between NP cells cultured from moderate and low grade IVD degeneration. The total number of DE lncRNAs identified in the present study was not as substantial as the 1,806 DE lncRNAs from NP tissues identified previously (33). The considerable difference the in number of identified DE lncRNAs may have occurred due to sample differences. First, the number of DE IncRNAs in NP tissues sourced from cases with high grade IVD degeneration is undoubtedly higher compared with that of tissues from patients with moderate IVD degeneration. Second, lncRNAs in NP tissues not only include lncRNAs from NP cells, but also those from certain inflammatory or immune cells gathered at IVD degenerative sites, as well as vesicles secreted from various cell types, which would hinder the study of lncRNA mechanisms as it is difficult to distinguish specific sources of lncRNAs. The 
A

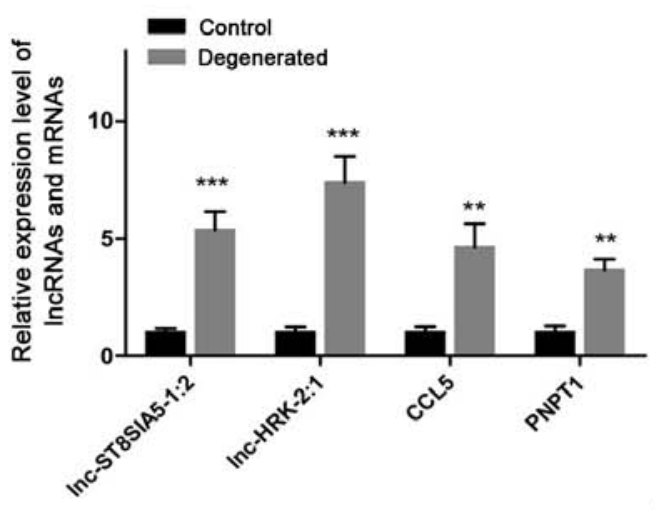

C

NC

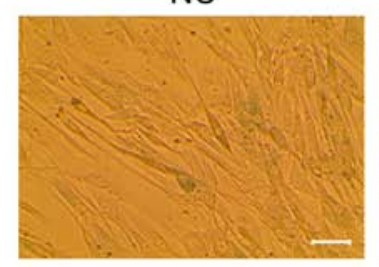

F

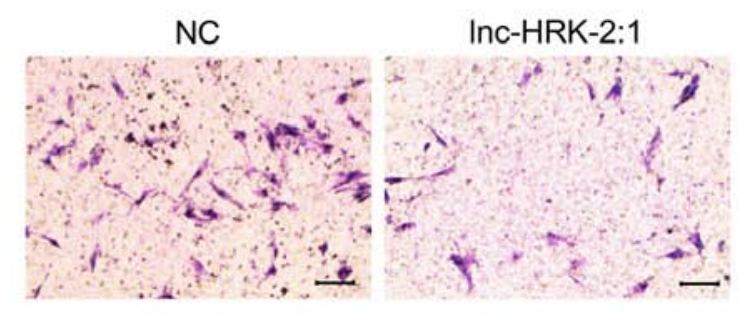

B

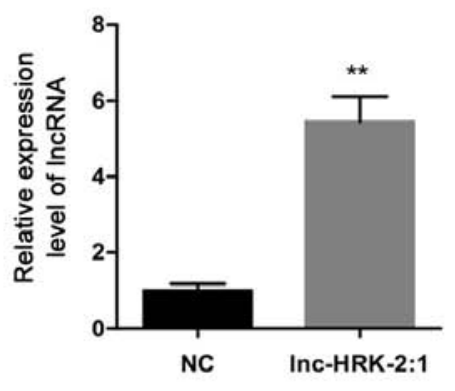

D

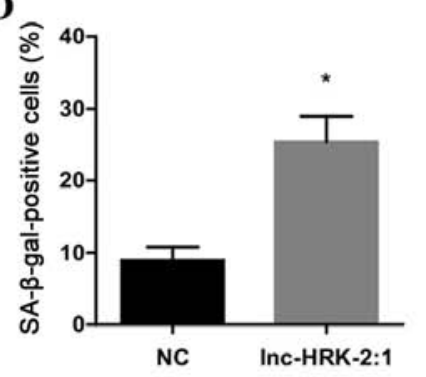

G

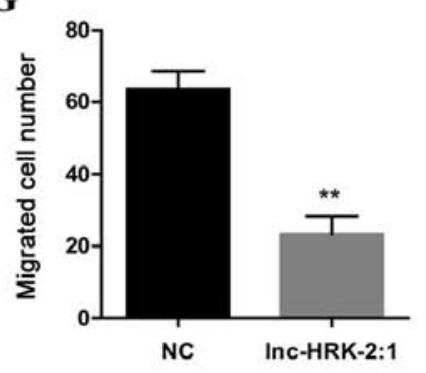

E

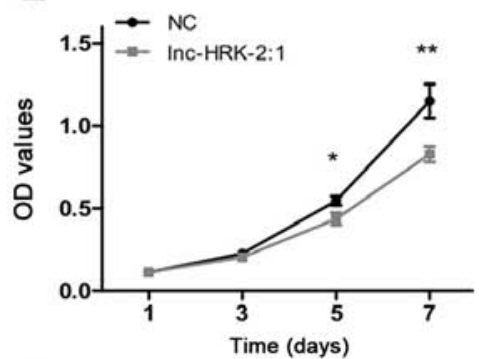

H

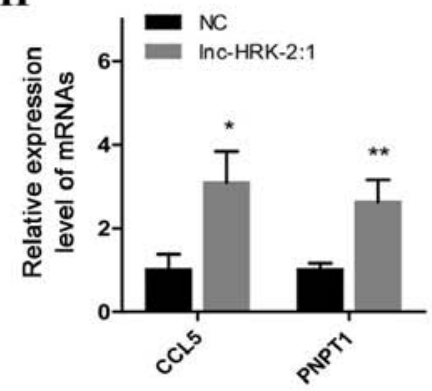

Figure 5. Overexpression of lnc-HRK-2:1 induces a senescent phenotype in control NP cells. (A) RT-qPCR analysis of the expression levels of two candidate lncRNAs, lnc-ST8SIA5-1:2 and lnc-HRK-2:1, and their targets CCL5 and PNPT1 in degenerated NP cells. (B) RT-qPCR analysis of the level of lnc-HRK-2:1 after overexpression of lnc-HRK-2:1 in control NP cells. (C and D) SA- $\beta$-gal staining analysis of senescent cells in lnc-HRK-2:1-overexpressing NP cells. Scale bar, $50 \mu \mathrm{m}$. (E) MTT assays were used to evaluate the role of lnc-HRK-2:1 on cell viability. (F and G) Transwell assays were performed to detect the role of lnc-HRK-2:1 in cell migratory ability. Scale bar, $100 \mu \mathrm{m}$. (H) RT-qPCR analysis of the expression levels of CCL5 and PNPT1 in control NP cells overexpressing lnc-HRK-2:1. " $\mathrm{P}<0.05,{ }^{* *} \mathrm{P}<0.01$ and ${ }^{* * * *} \mathrm{P}<0.001$ vs. control. NP, nucleus pulposus; OD, optical density; NC, negative control; RT-qPCR, reverse transcription-quantitative PCR; lnc-, long non-coding RNA; CCL5, C-C motif chemokine ligand 5; PNPT1, polyribonucleotide nucleotidyltransferase 1; SA- $\beta$-gal, senescence-associated $\beta$-galactosidase.

selection of NP cells rather than tissues as well as cases with moderate-rather than high-grade IVD degeneration may help to eliminate interference by other factors and enable the identification of IncRNAs involved in NP cellular phenotype alterations in early IVD degeneration.

LncRNA functions can be broadly classified into those that act on cis-targets, affecting the expression of neighboring genes, and those remote from transcription sites that operate on trans-targets $(23,32)$. In the present study, prediction tools identified 251 cis- and 2,170 trans-acting targets, and bioinformatics analyses provided an improved understanding of these targets. For example, proteasome subunit $\alpha 3$ (PSMA3), proteasome 26S subunit-2C non-ATPase 6 (PSMD6), inhibitor of DNA binding 2-2C HLH protein (ID2) and G2 and S-phase expressed 1 (GTSE1) were enriched in 'positive regulation of cell cycle arrest', whereas NSE4 homolog A-2C SMC5-SMC6 complex component (NSMCE4A), TP53 induced glycolysis regulatory phosphatase (TIGAR), acidic residue methyltransferase 1 (ARMT1), scaffolding protein involved in DNA repair
(SPIDR), family with sequence similarity 175 member A (FAM175A) and tumor protein-2C translationally-controlled 1 (TPT1) were enriched in 'regulation of response to DNA damage stimulus'. Cell cycle arrest as well as DNA damage are two important alterations identified as mechanisms involved when IVDs undergo aging and degeneration $(34,35)$. The majority of the targets identified in the present study were involved in either cell cycle arrest or DNA damage responses in other cells; however, the role of these targets in IVD degeneration is still unknown. Thus, these targets and their associated lncRNAs may provide potential choices for studies involving new targets and molecular mechanisms in IVD degeneration.

Next, cellular senescence-associated targets of lncRNAs with high FC (CCL5 and PNPT1, and their lncRNAs lnc-ST8SIA5-1:2 and lnc-HRK-2:1) were screened. RT-qPCR validation indicated that the two IncRNAs and two targets were all significantly upregulated in degenerative NP cells, which suggested that certain relationships existed between the 
two lncRNAs and increased cellular senescence in degenerated IVD NP cells. In addition, overexpression of lnc-HRK-2:1 in the control NP cells induced a senescent phenotype with an elevated percentage of senescent cells, and reduced growth and migratory abilities, and the expression levels of CCL5 and PNPT1 were also significantly enhanced, which indicated that lnc-HRK-2:1-mediated the senescence of NP cells is a key mechanism for the development of IVD degeneration.

CCL5, also termed 'regulated upon activation, normal $\mathrm{T}$ cell expressed and secreted', is a member of the pro-inflammatory chemokine family and serves a crucial role in immune cell chemotaxis (36). Although there is no previous evidence of association between CCL5 and cellular senescence in the process of IVD degeneration, elevated expression levels of CCL5 were detected in patients with degenerated IVD, discogram-positive painful IVD tissue, as well as normal NP cells treated with interleukin-1 $\beta$ compared with their corresponding controls (37-39). In addition, high levels of CCL5 secretion were also observed in aged theca-interstitial cells, senescent melanoma cells and a human fibroblast cell line (AG04382) from an aged donor $(40,41)$. Human polynucleotide phosphorylase (hPNPase $\mathrm{h}^{\text {old-35 }}$ ), the protein encoded by the PNPT1 gene, is an evolutionarily conserved 3',5'-exoribonuclease involved in regulating various physiological processes including the maintenance of mitochondrial homeostasis, aging-related inflammation and mitochondrial RNA import (42-44). Previous studies have demonstrated that overexpression of PNPT1 in cancer and normal cells results in distinctive growth inhibition with a characteristic senescent-like phenotype $(45,46)$. These results suggested that enhanced CCL5 and PNPT1 expression levels in NP cells from IVD degenerated samples as well as lnc-HRK-2:1 overexpressing cells in the present study maybe implicated in cellular senescence-associated IVD degeneration.

As demonstrated by the present microarray results, lnc-HRK-2:1 is located on chromosome 12 with a transcript size of 2,020 bp; reports regarding lnc-HRK-2:1 are lacking. The present study, for the first time, uncovered some of the roles of lnc-HRK-2:1 in IVD degeneration. CCL5 and PNPT1 were the predicted trans-targets of lnc-HRK-2:1, and overexpression of lnc-HRK-2:1 resulted in distinctive upregulation of the two targets, which may be due to direct trans-actions or indirect regulators. The first identified typical mechanism of trans-regulation was that involving the lncRNA HOTAIR acting as a scaffold that coordinates the recruitment of chromatin-modifying complexes to the HOXD locus, thus regulating gene expression (23), which provides an ideal basis for the future study of potential lnc-HRK-2:1 trans-acting mechanisms.

Imbalances in ECM metabolism serve an important role in IVD degeneration (47). To identify lncRNAs potentially involved in ECM metabolism, ECM-related GO and KEGG enrichment analyses were performed, and a total of 12 targets were observed in the GO term 'extracellular matrix organization'. Among these, ADAM metallopeptidase with thrombospondin type 1 motif 5 (ADAMTS5) and microfibrillar-associated protein 5 (MFAP5) were of interest. ADAMTS5 is one of the major proteolytic enzymes involved in ECM degradation and serves a vital role in this process $(48,49)$. MFAP5 is an ECM glycoprotein that has been demonstrated to be involved in ECM remodeling (50). Thus, it was inferred that lncRNAs corresponding to ADAMTS5 and MFAP5, NONHSAT081552 and lnc-MFAP5-3:2, may also serve certain roles in ECM metabolism during IVD degeneration.

There were certain limitations in the present study. First, the sample size (three samples per group) of NP tissues used for microarray analysis was small; an increased sample size may be beneficial for the identification of additional valuable lncRNAs. Second, the expression levels of lncHRK-2:1 have been confirmed at the cellular level, whereas the location and expression levels of lncHRK-2:1 in clinical IVD tissue is unclear, and further studies may clarify this.

In summary, the present study demonstrated that increased lnc-HRK-2:1 expression levels promoted the senescence of NP cells in the development of IVD degeneration, which may be attributed to the upregulation of CCL5 and PNPT1. In addition, hundreds of DE lncRNAs were identified, and thousands of potential DE lncRNA targets were predicted in the present study. The corresponding bioinformatic analyses conducted in the present study may also provide diverse perspectives to elucidate the mechanisms underlying IVD degeneration.

\section{Acknowledgements}

Not applicable.

\section{Funding}

This study was financially supported by the Guangxi Provincial Natural Science Foundation (grant no. JJBA40179).

\section{Availability of data and materials}

The datasets used and/or analyzed during the present study are available from the corresponding author on reasonable request.

\section{Authors' contributions}

FT and YW worked on the conception and design of the study. DL and DH analyzed and interpreted the data. JL, LL and HC performed the experiments and collected the data. All authors contributed to preparation of the manuscript. All authors read and approved the final manuscript.

\section{Ethics approval and consent to participate}

The present study was approved by the Ethics Review Board of Liuzhou Traditional Chinese Medicine Hospital, and written informed consent was obtained from all patients.

\section{Patient consent for publication}

Not applicable.

\section{Competing interests}

The authors declare that they have no competing interests. 


\section{References}

1. Wang F, Cai F, Shi R, Wang XH and Wu XT: Aging and age related stresses: A senescence mechanism of intervertebral disc degeneration. Osteoarthritis Cartilage 24: 398-408, 2016.

2. Katz JN: Lumbar disc disorders and low-back pain: Socioeconomic factors and consequences. J Bone Joint Surg Am 88 (Suppl 2): S21-S24, 2006.

3. Colombier P, Clouet J, Hamel O, Lescaudron L and Guicheux J: The lumbar intervertebral disc: From embryonic development to degeneration. Joint Bone Spine 81: 125-129, 2014.

4. Sampara P, Banala RR, Vemuri SK, Av GR and Gpv S: Understanding the molecular biology of intervertebral disc degeneration and potential gene therapy strategies for regeneration: A review. Gene Ther 25: 67-82, 2018.

5. Feng C, Liu H, Yang M, Zhang Y, Huang B and Zhou Y: Disc cell senescence in intervertebral disc degeneration: Causes and molecular pathways. Cell Cycle 15: 1674-1684, 2016.

6. Le Maitre CL, Freemont AJ and Hoyland JA: Accelerated cellular senescence in degenerate intervertebral discs: A possible role in the pathogenesis of intervertebral disc degeneration. Arthritis Res Ther 9: R45, 2007.

7. Gruber HE, Ingram JA, Norton HJ and Hanley EN Jr: Senescence in cells of the aging and degenerating intervertebral disc: Immunolocalization of senescence-associated beta-galactosidase in human and sand rat discs. Spine (Phila Pa 1976) 32: 321-327, 2007.

8. Kepler CK, Ponnappan RK, Tannoury CA, Risbud MV and Anderson DG: The molecular basis of intervertebral disc degeneration. Spine J 13: 318-330, 2013.

9. Cheng S, Li X, Lin L, Jia Z, Zhao Y, Wang D, Ruan D and Zhang Y: Identification of aberrantly expressed genes during aging in rat nucleus pulposus cells. Stem Cells Int 2019: 2785207, 2019.

10. Ben-Porath I and Weinberg RA: The signals and pathways activating cellular senescence. Int J Biochem Cell Biol 37: 961-976, 2005.

11. Brown CJ, Ballabio A, Rupert JL, Lafreniere RG, Grompe M, Tonlorenzi R and Willard HF: A gene from the region of the human $\mathrm{X}$ inactivation Centre is expressed exclusively from the inactive X chromosome. Nature 349: 38-44, 1991.

12. Derrien T, Johnson R, Bussotti G, Tanzer A, Djebali S, Tilgner H, Guernec G, Martin D, Merkel A, Knowles DG, et al: The GENCODE $v 7$ catalog of human long noncoding RNAs: Analysis of their gene structure, evolution, and expression. Genome Res 22: 1775-1789, 2012.

13. Cossu AM, Mosca L, Zappavigna S, Misso G, Bocchetti M, De Micco F, Quagliuolo L, Porcelli M, Caraglia M and Boccellino M: Long Non-coding RNAs as important biomarkers in laryngeal cancer and other head and neck tumours. Int J Mol Sci 20: 3444, 2019.

14. Zhang J, Wang P, Wan L, Xu S and Pang D: The emergence of noncoding RNAs as Heracles in autophagy. Autophagy 13: 1004-1024, 2017.

15. Li X, Lou Z, Liu J, Li H, Lei Y, Zhao X and Zhang F: Upregulation of the long noncoding RNA lncPolE contributes to intervertebral disc degeneration by negatively regulating DNA polymerase epsilon. Am J Transl Res 11: 2843-2854, 2019.

16. Wei R, Chen Y, Zhao Z, Gu Q and Wu J: LncRNA FAM83H-AS1 induces nucleus pulposus cell growth via targeting the Notch signaling pathway. J Cell Physiol 234: 22163-22171, 2019.

17. Xiao Y, Hu J and Yin W: Systematic Identification of Non-coding RNAs. Adv Exp Med Biol 1094: 9-18, 2018.

18. Pfirrmann CW, Metzdorf A, Zanetti M, Hodler J and Boos N: Magnetic resonance classification of lumbar intervertebral disc degeneration. Spine (Phila Pa 1976) 26: 1873-1878, 2001.

19. Tang X, Richardson WJ, Fitch RD, Brown CR, Isaacs RE and Chen J: A new non-enzymatic method for isolating human intervertebral disc cells preserves the phenotype of nucleus pulposus cells. Cytotechnology 66: 979-986, 2014.

20. Livak KJ and Schmittgen TD: Analysis of relative gene expression data using real-time quantitative PCR and the 2(-Delta Delta C(T)) method. Methods 25: 402-408, 2001.

21. Team RC: R: A language and environment for statistical computing. R Foundation for Statistical Computing, Vienna, Austria. Computing, 2013.

22. Petazzi P, Sandoval J, Szczesna K, Jorge OC, Roa L, Sayols S, Gomez A, Huertas D and Esteller M: Dysregulation of the long non-coding RNA transcriptome in a Rett syndrome mouse model. RNA Biol 10: 1197-1203, 2013.
23. Kopp F and Mendell JT: Functional classification and experimental dissection of long noncoding RNAs. Cell 172: 393-407, 2018.

24. Kent WJ, Sugnet CW, Furey TS, Roskin KM, Pringle TH, Zahler AM and Haussler D: The human genome browser at UCSC. Genome Res 12: 996-1006, 2002.

25. Ashburner M, Ball CA, Blake JA, Botstein D, Butler $\mathrm{H}$, Cherry JM, Davis AP, Dolinski K, Dwight SS, Eppig JT, et al: Gene ontology: Tool for the unification of biology. The Gene Ontology Consortium. Nat Genet 25: 25-29, 2000.

26. Kanehisa M and Goto S: KEGG: Kyoto encyclopedia of genes and genomes. Nucleic Acids Res 28: 27-30, 2000.

27. Zhang YG, Sun ZM, Liu JT, Wang SJ, Ren FL and Guo X: Features of intervertebral disc degeneration in rat's aging process. J Zhejiang Univ Sci B 10: 522-527, 2009.

28. Khan AA, Mahmood S, Saif T and Gul A: Spinal cord injury without radiographic abnormality (SCIWORA) in adults: A report of two cases. J Pak Med Assoc 67: 1275-1277, 2017.

29. Amelot A, Bouazza S, George B, Orabi M and Bresson D: Anterior extrusion of fusion cage in posttraumatic cervical disk disease. J Neurol Surg A Cent Eur Neurosurg 76: 168-171, 2015.

30. Zhao CQ, Wang LM, Jiang LS and Dai LY: The cell biology of intervertebral disc aging and degeneration. Ageing Res Rev 6: 247-261, 2007.

31. Jeong SW, Lee JS and Kim KW: In vitro lifespan and senescence mechanisms of human nucleus pulposus chondrocytes. Spine J 14: 499-504, 2014.

32. Sun M and Kraus WL: From discovery to function: The expanding roles of long noncoding RNAs in physiology and disease. Endocr Rev 36: 25-64, 2015.

33. Wan ZY, Song F, Sun Z, Chen YF, Zhang WL, Samartzis D, Ma CJ, Che L, Liu X, Ali MA, et al: Aberrantly expressed long noncoding RNAs in human intervertebral disc degeneration: A microarray related study. Arthritis Res Ther 16: 465, 2014.

34. Zhan S, Wang K, Song Y, Li S, Yin H, Luo R, Liao Z, Wu X, Zhang $\mathrm{Y}$ and Yang C: Long non-coding RNA HOTAIR modulates intervertebral disc degenerative changes via Wnt/ $\beta$-catenin pathway. Arthritis Res Ther 21: 201, 2019.

35. Vo NV, Hartman RA, Patil PR, Risbud MV, Kletsas D, Iatridis JC, Hoyland JA, Le Maitre CL, Sowa GA and Kang JD: Molecular mechanisms of biological aging in intervertebral discs. J Orthop Res 34: 1289-1306, 2016.

36. Pattappa G, Peroglio M, Sakai D, Mochida J, Benneker LM, Alini M and Grad S: CCL5/RANTES is a key chemoattractant released by degenerative intervertebral discs in organ culture. Eur Cell Mater 27: 124-136, 2014.

37. Kepler CK, Markova DZ, Dibra F, Yadla S, Vaccaro AR, Risbud MV, Albert TJ and Anderson DG: Expression and relationship of proinflammatory chemokine RANTES/CCL5 and cytokine IL-1 $\beta$ in painful human intervertebral discs. Spine (Phila Pa 1976) 38: 873-880, 2013.

38. Grad S, Bow C, Karppinen J, Luk KD, Cheung KM, Alini M and Samartzis D: Systemic blood plasma CCL5 and CXCL6: Potential biomarkers for human lumbar disc degeneration. Eur Cell Mater 31: 1-10, 2016.

39. Liu W, Liu D, Zheng J, Shi P, Chou PH, Oh C, Chen D, An HS and Chee A: Annulus fibrosus cells express and utilize C-C chemokine receptor 5 (CCR5) for migration. Spine J 17: 720-726, 2017.

40. Shen L, Chen Y, Cheng J, Yuan S, Zhou S, Yan W, Liu J, Luo A and Wang S: CCL5 secreted by senescent theca-interstitial cells inhibits preantral follicular development via granulosa cellular apoptosis. J Cell Physiol 234: 22554-22564, 2019.

41. Eyman D, Damodarasamy M, Plymate SR and Reed MJ: CCL5 secreted by senescent aged fibroblasts induces proliferation of prostate epithelial cells and expression of genes that modulate angiogenesis. J Cell Physiol 220: 376-381, 2009.

42. Sarkar D and Fisher PB: Polynucleotide phosphorylase: An evolutionary conserved gene with an expanding repertoire of functions. Pharmacol Ther 112: 243-263, 2006.

43. Sarkar D, Lebedeva IV, Emdad L, Kang DC, Baldwin AS Jr and Fisher PB: Human polynucleotide phosphorylase (hPNPaseold-35): A potential link between aging and inflammation. Cancer Res 64: 7473-7478, 2004.

44. Sokhi UK, Das SK, Dasgupta S, Emdad L, Shiang R, DeSalle R, Sarkar D and Fisher PB: Human polynucleotide phosphorylase (hPNPaseold-35): Should I eat you or not-that is the question? Adv Cancer Res 119: 161-190, 2013. 
45. Sarkar D, Leszczyniecka M, Kang DC, Lebedeva IV, Valerie K, Dhar S, Pandita TK and Fisher PB: Down-regulation of Myc as a potential target for growth arrest induced by human polynucleotide phosphorylase (hPNPaseold-35) in human melanoma cells. J Biol Chem 278: 24542-24551, 2003.

46. Chan I, Lebedeva IV, Su ZZ, Sarkar D, Valerie K and Fisher PB: Progression elevated gene-3 promoter (PEG-Prom) confers cancer cell selectivity to human polynucleotide phosphorylase (hPNPase(old-35))-mediated growth suppression. J Cell Physiol 215: 401-409, 2008.

47. Wang WJ, Yu XH, Wang C, Yang W, He WS, Zhang SJ, Yan YG and Zhang J: MMPs and ADAMTSs in intervertebral disc degeneration. Clin Chim Acta 448: 238-246, 2015.

48. Banala RR, Vemuri SK, Dar GH, Palanisamy V, Penkulinti M, Surekha MV, Gurava Reddy AV, Nalam MR and Subbaiah G: Efficiency of dual siRNA-mediated gene therapy for intervertebral disc degeneration (IVDD). Spine J 19: 896-904, 2019.
49. Lu L, Hu J, Wu Q, An Y, Cui W, Wang J and Ye Z: Berberine prevents human nucleus pulposus cells from IL1- $\beta$-induced extracellular matrix degradation and apoptosis by inhibiting the NF- $\kappa B$ pathway. Int J Mol Med 43: 1679-1686, 2019.

50. Vaittinen $M$, Kolehmainen M, Ryden M, Eskelinen $M$, Wabitsch M, Pihlajamaki J, Uusitupa M and Pulkkinen L: MFAP5 is related to obesity-associated adipose tissue and extracellular matrix remodeling and inflammation. Obesity (Silver Spring) 23: 1371-1378, 2015.

(i) (9) This work is licensed under a Creative Commons Attribution-NonCommercial-NoDerivatives 4.0 International (CC BY-NC-ND 4.0) License. 